\title{
Sensitivity analysis of dimensionless parameters for physical simulation of water-flooding reservoir
}

\author{
BAI Yuhu, LI Jiachun \& ZHOU Jifu
}

Division of Engineering Sciences, Institute of Mechanics, Chinese Academy of Sciences, Beijing 100080 , China

Correspondence should be addressed to Bai Yuhu (email: byh21 @ sina.com)

Received July 15, 2004

\begin{abstract}
A numerical approach to optimize dimensionless parameters of water-flooding porous media flows is proposed based on the analysis of the sensitivity factor defined as the variation ration of a target function with respect to the variation of dimensionless parameters. A complete set of scaling criteria for water-flooding reservoir of five-spot well pattern case is derived from the 3-D governing equations, involving the gravitational force, the capillary force and the compressibility of water, oil and rock. By using this approach, we have estimated the influences of each dimensionless parameter on experimental results, and thus sorting out the dominant ones with larger sensitivity factors ranging from $10^{-4}$ to $10^{0}$.
\end{abstract}

Keywords: sensitivity analysis, model scaling, numerical experiment, water-flooding, two-phase flow.

DOI: 10.1360/04ye0290

Petroleum industry in China is confronting challenge in oil supply. On the one hand, the total storage is hardly to meet emergent demands. On the other hand, the oil fields developed in early years are costly operated due to the high average water ratio even up to $80 \%$. The lack in energy resource considerably promotes the enhancement of oil recovery. Experts ${ }^{[1]}$ estimated that with the oil recovery enhanced by $1 \%$, the oil production would be augmented as much as the annual total output with 150 billion Yuan profit. Therefore, a better understanding in the behavior of water, oil and gas underground is indispensable.

Physical simulation is an important approach to reveal mechanisms of porous media flows for design and optimization of development programs. Compared with field tests, this approach seems to be cheaper, time-saving and easier to implement. The principle of similarity or scaling law is crucial for physical simulations ${ }^{[2]}$. Geertsma et al.$^{[3]}$ derived the scaling criteria for flows in cold water, heated water and solvent flooding reservoir 
by using both inspectional and dimensional analysis methods, showing that the effect of inertia force could be neglected in most circumstances. In the heated water-flooding reservoir, the effect of the gravitational force can be scaled with porous medium parameters relaxed. However, the pressure drop, the capillary force, the residual oil saturation and relative permeability cannot be well modeled. Kimber et al. ${ }^{[4]}$ obtained a set of scaling criteria for steam and steam additive recovery reservoir. By relaxing the requirement of geometric similarity, we may employ the same fluids and porous medium in the models as in the prototypes. Islam et al $^{[5]}$ got the scaling criteria for polymer, emulsion and foam flooding experiments by the inspectional analysis. They focused on the complicated flooding processes involving mass transfer among phases, the interfacial tension, diffusion, dispersion, absorption and mechanical entrapment. They argued that it is necessary to relax the geometric similarity in order to properly scale dispersion and pressure-induced effects in some circumstances. Islam et al. ${ }^{[6]}$ gained the scaling criteria of surfactant-enhanced alkaline/polymer multiple flooding flows, which involve transient interfacial tension and non-equilibrium mass transfer phenomena. They have observed that in most cases the mass transfer rate in the model is different from that in the prototype. However, both the rates have been assumed the same in previous studies. Shen et al. ${ }^{[7]}$ assumed that the ratios of the gravitational and the driving forces to the capillary force were essential similarity parameters for water-flooding reservoirs. Generally speaking, there are so many parameters involved in the process of water driving oil flows due to the complex mechanisms. Therefore, it is very difficult or sometimes even impossible to keep all the similarity parameters identical in the laboratory experiment. Pozzi and Blackwell ${ }^{[8]}$ pointed out that precise scaling of transverse dispersion coupled with the requirement of geometric similarity would impractically require a large model and a very long time interval for experiments. In particular, some dimensionless parameters are contradictory. To tackle this kind of problems, the efficient and practical way out is to single out the dominant parameters and to relax the secondary ones in laboratory experiments. It is not easy or even impossible to theoretically arrange all the dimensionless parameters in the orders of importance. Previous literatures at most have provided the importance of part of the dimensionless parameters qualitatively ${ }^{[9]}$. Therefore, how to quantify the dominant degree among them is still a problem open to us.

The authors of the present paper have suggested a numerical approach of sensitivity analysis of dimensionless parameters. To begin with, we have defined the sensitivity factor in section 1 . Then, we have derived a set of scaling criteria of water-flooding reservoir flows, accounting for the gravitational and capillary forces, and the compressibility of fluids and rock, and then validated the code in sections 2 and 3, respectively. Finally, we have quantified the sensitivity of all the dimensionless parameters by using the proposed numerical approach. According to the values of sensitivity factors we may select the dominant dimensionless parameters that should be satisfied in laboratory experiments. 


\section{Sensitivity analysis of dimensionless parameters}

Actually, it is not realistic for us to estimate the role of each dimensionless parameter on experimental results by physical simulation. In contrast, we present a numerical approach to evaluate the effect of each dimensionless parameter on a target function. The sensitivity factor of a given dimensionless parameter $\pi_{i}$ is firstly defined as follows:

$$
S_{i}=\frac{\partial\left[f\left(\pi_{1}, \pi_{2}, \cdots, \pi_{N}\right) / f_{\mathrm{p}}\right]}{\partial\left(\pi_{i} / \pi_{i \mathrm{p}}\right)}(i=1,2, \cdots, N)
$$

where $f\left(\pi_{1}, \pi_{2}, \cdots, \pi_{N}\right)$ denotes a target function concerned in the experiment. $S_{i}$ is called sensitivity factor meaning the relative variation ration of the target function with respect to that of a dimensionless parameter. In a water-flooding experiment, the target function can be expressed as

$$
f\left(\pi_{1}, \pi_{2}, \cdots, \pi_{N}\right)=\int_{0}^{T_{\mathrm{D}}} \eta\left(\pi_{1}, \pi_{2}, \cdots, \pi_{N}, t_{\mathrm{D}}\right) \mathrm{d} t_{\mathrm{D}},
$$

where $\eta\left(\pi_{1}, \pi_{2}, \cdots, \pi_{N}, t_{\mathrm{D}}\right)$ represents the oil recovery curve as shown in Fig. 1, and $T_{\mathrm{D}}$ the dimensionless time span of development. In our numerical scheme, the sensitivity factor is written in the following difference form

$$
S_{i}=\frac{\Delta a_{i \mathrm{~m}} / a_{0}}{\left|w_{i}\right|}
$$

in which $w_{i}=\frac{\pi_{i \mathrm{~m}}-\pi_{i \mathrm{p}}}{\pi_{i \mathrm{p}}}$ stands for the relative distortion of the $i$ th dimensionless parameter, $a_{0}=\int_{0}^{T_{\mathrm{D}}} \eta_{\mathrm{p}}\left(\pi_{1 \mathrm{p}}, \pi_{2 \mathrm{p}}, \cdots, \pi_{N \mathrm{p}}, t_{\mathrm{D}}\right) \mathrm{d} t_{\mathrm{D}}$ is the area under the oil recovery curve of

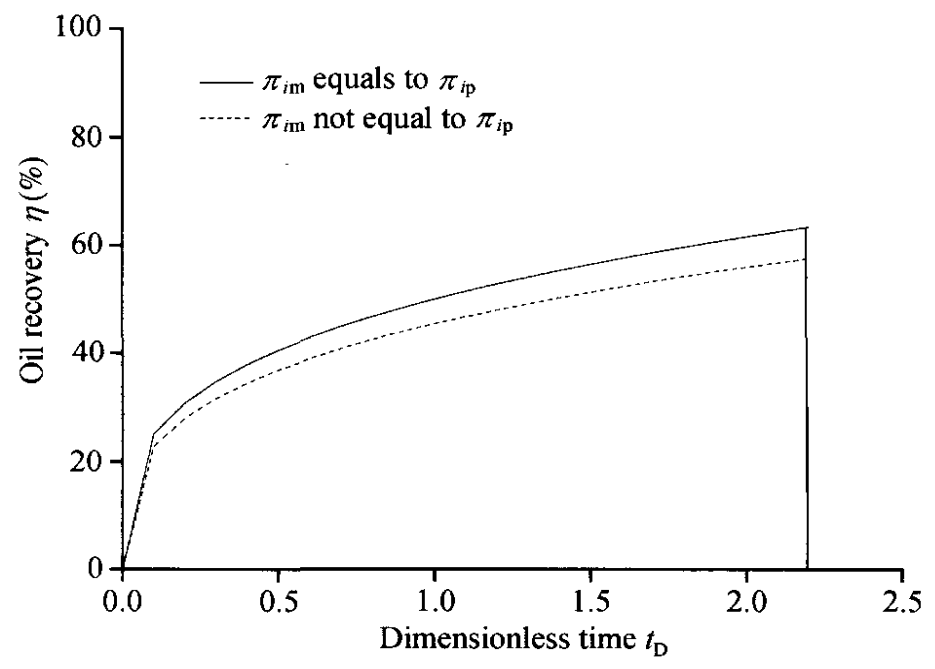

Fig. 1. The sketch of the comparison of oil recovery between a fully similar model or a prototype and partially similar model, in which the $i$ th dimensionless parameter is given as a small deviation from the prototype. 
the prototype, $\Delta a_{i \mathrm{~m}}=\int_{0}^{T_{\mathrm{D}}}\left|\eta_{\mathrm{m}}\left(\pi_{1 \mathrm{~m}}, \pi_{2 \mathrm{~m}}, \cdots, \pi_{i \mathrm{~m}}, \cdots, \pi_{N \mathrm{~m}}, t_{\mathrm{D}}\right)-\eta_{\mathrm{p}}\left(\pi_{1 \mathrm{p}}, \pi_{2 \mathrm{p}}, \cdots, \pi_{N \mathrm{p}}, t_{\mathrm{D}}\right)\right| \mathrm{d} t_{\mathrm{D}}$ means the deviation of the oil recovery of the model from the prototype. One curve applies to the situation, in which the $i$ th dimensionless parameter of the model deviates from the prototype, and the other to the fully similar model. Subscripts $\mathrm{m}$ and $\mathrm{p}$ indicate the model and the reservoir prototype, respectively. Arranging the sensitivity factor $S_{i}$ in order, we can conveniently exhibit the importance degree of each dimensionless parameter. In the following sections, we take the similarity of water-flooding reservoir as an example to demonstrate the procedure.

\section{Governing equations and solution procedure}

Let us consider two-phase porous media flows of water and oil. Suppose the flow obey Darcy's law. Taking into consideration the fluids of constant viscosity, the capillary and gravitational forces and the compressibility of fluids and rock, the governing equations are as follows:

$$
\begin{gathered}
\nabla \cdot\left(\frac{\rho_{\mathrm{o}} K_{\mathrm{o}}}{\mu_{\mathrm{o}}} \nabla\left(p+\rho_{\mathrm{o}} g \nabla z\right)\right)+Q_{\mathrm{o}}=\frac{\partial\left(\phi \rho_{\mathrm{o}} s_{\mathrm{o}}\right)}{\partial t}, \\
\nabla \cdot\left(\frac{\rho_{\mathrm{w}} K_{\mathrm{w}}}{\mu_{\mathrm{w}}} \nabla\left(p+\rho_{\mathrm{w}} g \nabla z\right)\right)+Q_{\mathrm{w}}=\frac{\partial\left(\phi \rho_{\mathrm{w}} s_{\mathrm{w}}\right)}{\partial t}, \\
Q_{\mathrm{o}}=\frac{\pi \rho_{\mathrm{o}} k_{\mathrm{o}}\left(p_{\mathrm{wf}}-p_{\mathrm{o}}\right)}{2 \mu_{\mathrm{o}} \ln \left(r_{\mathrm{eo}} / r_{\mathrm{o}}\right)} \delta\left(x-x_{\mathrm{p}}\right) \delta\left(y-y_{\mathrm{p}}\right), \\
Q_{\mathrm{w}}=\frac{\rho_{\mathrm{w}} q_{\mathrm{I}}}{4 h} \delta(x) \delta(y)+\frac{\pi \rho_{\mathrm{w}} k_{\mathrm{w}}\left(p_{\mathrm{wf}}-p_{\mathrm{w}}\right)}{2 \mu_{\mathrm{w}} \ln \left(r_{\mathrm{eo}} / r_{\mathrm{o}}\right)} \delta\left(x-x_{\mathrm{p}}\right) \delta\left(y-y_{\mathrm{p}}\right),
\end{gathered}
$$

where $p, \mu, \rho, K$, and $s$ mean pressure, density, viscosity, effective permeability and saturation, subscript $\mathrm{w}$ and $\mathrm{o}$ indicate water and oil phases, respectively. And $p_{\mathrm{c}}$ is the capillary force, $\phi$ the porosity of rock, $g$ the gravitational acceleration, $\left(x_{p}, y_{p}\right)$ the coordinate of production well, $q_{\mathrm{I}}$ the injection rate per unit thickness and $r_{\mathrm{o}}$ the well radius.

The constitutive equations are

$$
\begin{gathered}
\rho_{\mathrm{o}}=\rho_{\mathrm{o} 0}\left(1+C_{\mathrm{o}}\left(p_{\mathrm{o}}+\rho_{\mathrm{o}} g z-p_{\mathrm{o} 0}\right)\right), \\
\rho_{\mathrm{w}}=\rho_{\mathrm{w} 0}\left(1+C_{\mathrm{w}}\left(p_{\mathrm{w}}+\rho_{\mathrm{w}} g z-p_{\mathrm{w} 0}\right)\right), \\
\phi=\phi_{0}\left(1+C_{\phi}\left(\frac{p_{\mathrm{w}}+p_{\mathrm{o}}+\rho_{\mathrm{o}} g z+\rho_{\mathrm{w}} g z}{2}-\frac{p_{\mathrm{w} 0}+p_{\mathrm{o} 0}}{2}\right)\right),
\end{gathered}
$$

in which the symbols have the same meaning as the above. Subscript 0 indicates physical quantities at a certain condition, $C_{\mathrm{o}}, C_{\mathrm{w}}$ and $C_{\phi}$ the compressibility of oil, water and rock, respectively. 
The capillary force equation can be expressed as

$$
p_{\mathrm{c}}=p_{\mathrm{o}}-p_{\mathrm{w}}=\sigma \cos \theta \sqrt{\phi / K} J\left(s_{\mathrm{w}}\right),
$$

where $K$ denotes the absolute permeability, $\sigma$ the interfacial tension at the interface of water and oil phases, $\theta$ the contact angle between water and oil phases, and $J\left(s_{\mathrm{w}}\right)$ the capillary force function.

The saturation relation reads

$$
s_{\mathrm{o}}+s_{\mathrm{w}}=1
$$

Initial conditions are given as

$$
\begin{aligned}
& p_{\mathrm{o}} \mathrm{l}_{t=0}=p_{\mathrm{oi}}, \\
& \left.s_{\mathrm{w}}\right|_{t=0}=s_{\mathrm{wi}},
\end{aligned}
$$

and boundary conditions are

$$
\frac{\partial p_{l}}{\partial x}=0, \quad \frac{\partial p_{l}}{\partial y}=0, \quad \frac{\partial p_{l}}{\partial z}+\rho_{l} g=0 \quad l=0, w
$$

The constitutive equations can be further simplified. According to

$$
\begin{aligned}
\rho_{\mathrm{o}} & =\rho_{\mathrm{o} 0}\left(1+C_{\mathrm{o}}\left(p_{\mathrm{o}}+\rho_{\mathrm{o}} g z-p_{\mathrm{o} 0}\right)\right) \\
& =\rho_{\mathrm{o} 0}\left(1+C_{\mathrm{o}}\left(p_{\mathrm{o}}+\rho_{\mathrm{o} 0}\left(1+C_{\mathrm{o}}\left(p_{\mathrm{o}}+\rho_{\mathrm{o}} g z-p_{\mathrm{o} 0}\right)\right) g z-p_{\mathrm{o} 0}\right)\right),
\end{aligned}
$$

omitting the term $C_{\mathrm{o}}^{2}\left(p_{\mathrm{o}}+\rho_{\mathrm{o}} g z-p_{\mathrm{o} 0}\right)$, eq. (8) can be reduced to

$$
\rho_{\mathrm{o}}=\rho_{\mathrm{o} 0}\left(1+C_{\mathrm{o}}\left(p_{\mathrm{o}}+\rho_{\mathrm{o} 0} g z-p_{\mathrm{o} 0}\right)\right) \text {. }
$$

Analogously, we have

$$
\begin{gathered}
\rho_{\mathrm{w}}=\rho_{\mathrm{w} 0}\left(1+C_{\mathrm{w}}\left(p_{\mathrm{w}}+\rho_{\mathrm{w} 0} g z-p_{\mathrm{w} 0}\right)\right) \\
\phi=\phi_{0}\left(1+C_{\phi}\left(\frac{p_{\mathrm{w}}+p_{\mathrm{o}}+\rho_{\mathrm{o} 0} g z+\rho_{\mathrm{w} 0} g z}{2}-\frac{p_{\mathrm{w} 0}+p_{\mathrm{o} 0}}{2}\right)\right) .
\end{gathered}
$$

The governing equations are discretized with the finite difference method and solved by the implicit pressure-explicit saturation method ${ }^{[11]}$. The discrete implicit oil pressure equation turns out:

$$
\begin{aligned}
& a_{i, j, k} p_{\mathrm{o} i-1, j, k}+b_{i, j, k} p_{\mathrm{o} i+1, j, k}+c_{i, j, k} p_{\mathrm{o} i, j-1, k}+d_{i, j, k} p_{\mathrm{o} i, j+1, k} \\
& +e_{i, j, k} p_{\mathrm{o} i, j, k-1}+f_{i, j, k} p_{\mathrm{o} i, j, k+1}+g_{i, j, k} p_{\mathrm{o} i, j, k}=h_{i, j, k},
\end{aligned}
$$

where 


$$
\begin{aligned}
& a_{i, j, k}=a_{\mathrm{o} i, j, k}+A a_{\mathrm{w} i, j, k}, \quad b_{i, j, k}=b_{\mathrm{o} i, j, k}+A b_{\mathrm{w} i, j, k}, \\
& c_{i, j, k}=c_{\mathrm{o} i, j, k}+A c_{\mathrm{w} i, j, k}, \quad d_{i, j, k}=d_{\mathrm{o} i, j, k}+A d_{\mathrm{w} i, j, k}, \\
& e_{i, j, k}=e_{\mathrm{o} i, j, k}+A e_{\mathrm{w} i, j, k}, \quad f_{i, j, k}=f_{\mathrm{o} i, j, k}+A f_{\mathrm{w} i, j, k}, \quad g_{i, j, k}=g_{\mathrm{o} i, j, k}+A g_{\mathrm{w} i, j, k}, \\
& h_{i, j, k}=h_{\mathrm{o} i, j, k}+A h_{\mathrm{w} i, j, k}+A\left(c_{\mathrm{w} i, j, k} p_{\mathrm{c} i, j-1, k}+a_{\mathrm{w} i, j, k} p_{\mathrm{c} i-1, j, k}+b_{\mathrm{w} i, j, k} p_{\mathrm{c} i+1, j, k}\right. \\
& \left.+d_{\mathrm{w} i, j, k} p_{\mathrm{c} i, j+1, k}+e_{\mathrm{w} i, j, k} p_{\mathrm{c} i, j, k-1}+f_{\mathrm{w} i, j, k} p_{\mathrm{c} i, j, k+1}+g_{\mathrm{w} i, j, k} p_{\mathrm{c} i, j, k}\right) \text {, } \\
& a_{l i, j, k}=\frac{2 \lambda_{l x i-\frac{1}{2}}}{\left(\Delta x_{i-1}+\Delta x_{i}\right) \Delta x_{i-1}}, \quad b_{l i, j, k}=\frac{2 \lambda_{l x i+\frac{1}{2}}}{\left(\Delta x_{i}+\Delta x_{i-1}\right) \Delta x_{i}}, \quad c_{l i, j, k}=\frac{2 \lambda}{\left(\Delta y_{j-1}+\Delta y_{j}\right) \Delta y_{j-1}}, \\
& d_{l i, j, k}=\frac{2 \lambda}{\left(\Delta y_{j}+\Delta y_{j-1}\right) \Delta y_{j}}, \quad e_{l i, j, k}=\frac{2 \lambda}{\left(\Delta z_{k-1}+\Delta z_{k}\right) \Delta z_{k-1}}, \quad f_{l i, j, k}=\frac{2 \lambda}{\left(\Delta z_{k-1}+\Delta z_{k}\right) \Delta z_{k}}, \\
& g_{l i, j, k}=a_{l i, j, k}+b_{l i, j, k}+c_{l i, j, k}+d_{l i, j, k}+e_{l i, j, k}+f_{l i, j, k}+s_{l i, j, k}\left(\phi_{i, j, k} \rho_{l 0} C_{l}+\rho_{l i, j, k} \phi_{0} C_{\phi}\right) \text {, } \\
& h_{l i, j, k}=-\rho_{l i, j, k} q_{l}-\frac{2\left(\lambda_{l z k+\frac{1}{2}} \rho_{l_{i, j, k+\frac{1}{2}}} g-\lambda_{l z k-\frac{1}{2}} \rho_{l i, j, k-\frac{1}{2}} g\right)}{\Delta z_{k-1}+\Delta z_{k}} \\
& -s_{l i, j, k}\left(\phi_{i, j, k} \rho_{l 0} C_{l}+\rho_{l i, j, k} \phi_{0} C_{\phi}\right) \frac{p_{l i, j, k}^{n}}{\Delta t^{n}}
\end{aligned}
$$

in which $\lambda_{l}=\rho_{l} K K_{r l} / \mu_{l}$ denotes mobility coefficient, $l=\mathrm{o}, \mathrm{w}, \quad A=\rho_{\mathrm{o}} / \rho_{\mathrm{w}}$.

Solving eq. (19) leads to the solution of oil phase pressure. Then we can explicitly obtain oil saturation by substituting the oil pressure into eq. (4). In order to improve the accuracy and reduce the mass conservation error, we divide the time step for solving oil pressure into several small steps. In each small step, the saturation equation is solved explicitly. The mobility coefficient takes the harmonic mean between the neighboring grids for solving oil pressure equation. For example, the mobility coefficient in $x$ direction is

$$
\lambda_{i+\frac{1}{2}, j, k}=\frac{\Delta x_{i+1}+\Delta x_{i}}{\Delta x_{i+1} / \lambda_{i+1, j, k}+\Delta x_{i} / \lambda_{i, j, k}},
$$

so are those in $y$ and $z$ directions. When solving oil saturation, the mobility coefficient takes the value at the upstream node, accounting for the lag variation of the oil saturation in the flow direction.

The validation of the code is made by comparing the numerical results with the theory of Buckley-Leverett ${ }^{[12]}$. The assumptions for Buckley-leverett equation are: one-dimensional flow occurring in a homogenous constant thickness rock layer, the negligible cap- 
illary and gravitational forces, the compressibility of fluids and porous medium being not considered, and the constant density and viscosity of fluids. So the position of a given water saturation $x\left(s_{\mathrm{w}}, t\right)$ at time $t$ is determined by the following expression:

$$
x\left(s_{\mathrm{w}}, t\right)=x\left(s_{\mathrm{w}}, 0\right)+\frac{f^{\prime}\left(s_{\mathrm{w}}\right)}{A \phi} \int_{0}^{t} Q(t) \mathrm{d} t,
$$

in which $x\left(s_{\mathrm{w}}, 0\right)$ represents the initial distribution of water saturation, $Q(t)$ the injection rate, $\phi$ the porosity, $A$ the cross section area of the reservoir, and $f^{\prime}\left(s_{w}\right)$ the derivative of the water ratio to the water saturation. Fig. 2 shows the comparison of the displacements of water saturation contours between the present numerical results and the solution of Buchley-Leverett equation at $t=3000 \mathrm{~s}$ and $t=7000 \mathrm{~s}$, respectively. In Fig. 3 plotted is the evolution of water saturation at the two positions of $x=10 \mathrm{~m}$ and $x=15 \mathrm{~m}$ compared with the theory of Buchley-Leverett. It is seen that both the spatial distribution and time evolution of the water saturation agree well with the theoretical results, which completes the validation of the numerical code employed.

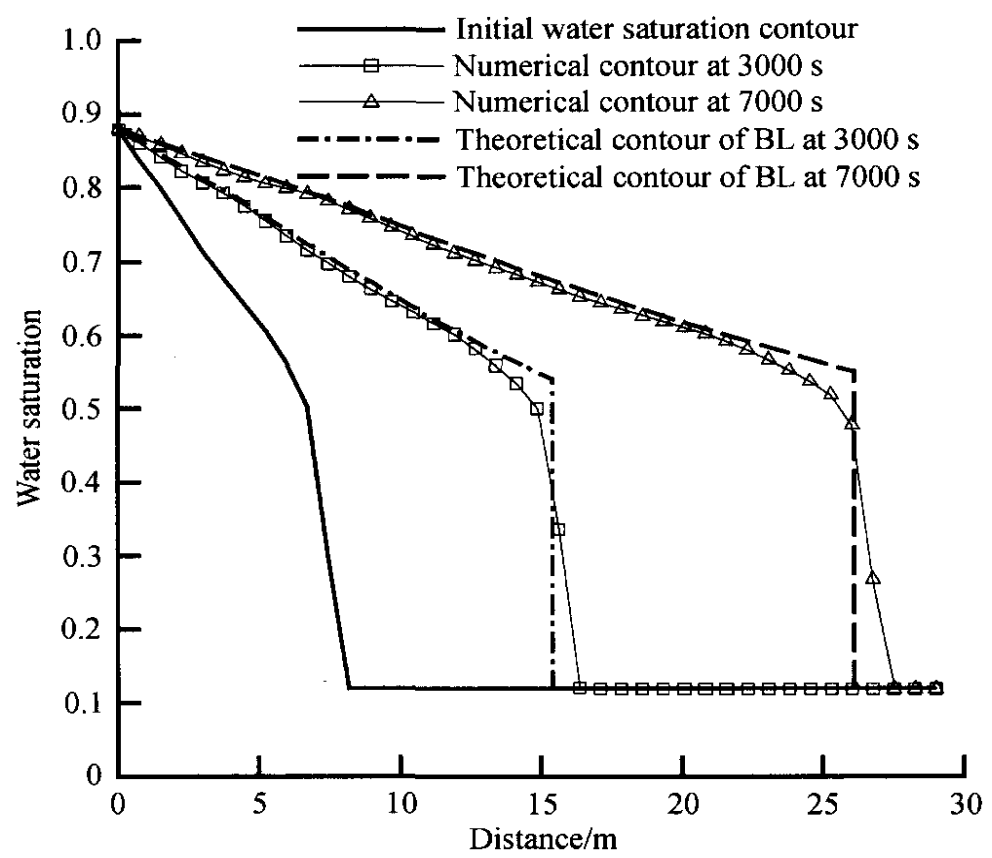

Fig. 2. Comparison of the displacement of iso-water saturation contours between theoretical and numerical results at the time of $t=3000 \mathrm{~s}$ and $7000 \mathrm{~s}$.

\section{Similarity criteria}

Consider a quarter of three-dimensional five-spot well pattern of water-flooding reservoir with its length of $l$, width of $w$, and thickness of $h$. The production and injection wells locate at the end of a diagonal, their horizontal coordinates being $(0,0)$ and 


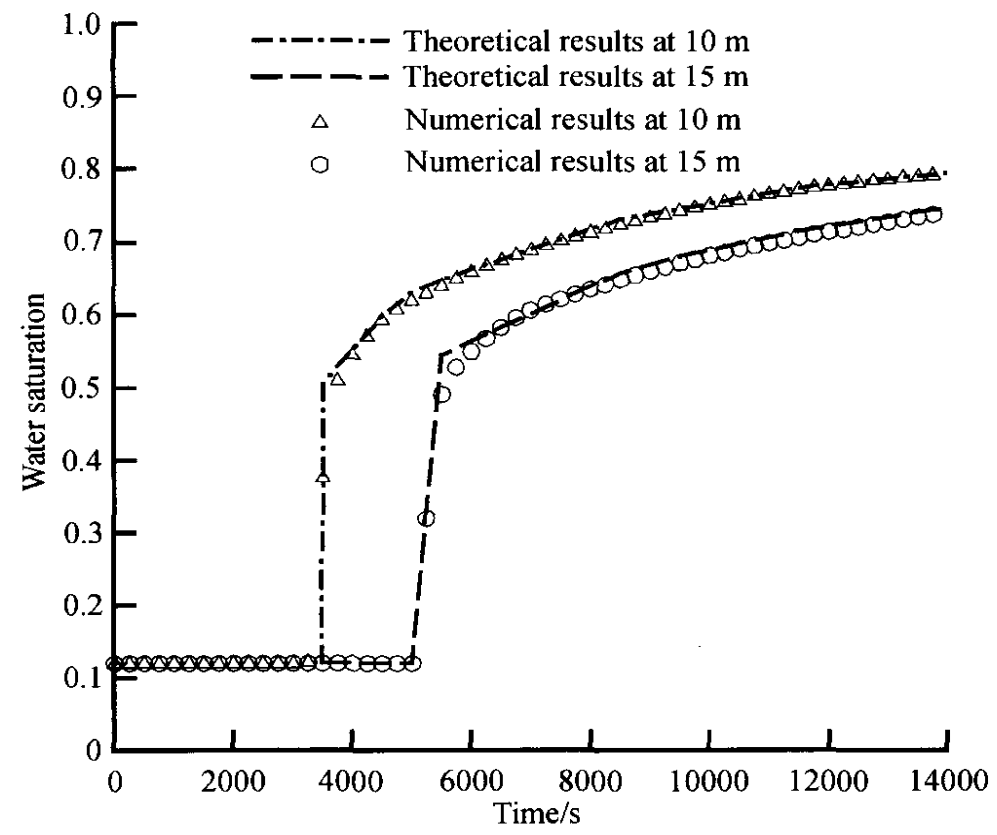

Fig. 3. Comparison of water saturation vs time between theoretical and numerical results at the positions of $x=10$ $\mathrm{s}$ and $15 \mathrm{~m}$.

$\left(x_{\mathrm{p}}, y_{\mathrm{p}}\right)$, respectively. To derive the similarity criteria, we introduce the dimensionless independent variables:

$$
x_{D}=\frac{x}{x_{\mathrm{R}}}, y_{D}=\frac{y}{y_{\mathrm{R}}}, z_{D}=\frac{z}{z_{\mathrm{R}}}, t_{D}=\frac{q_{\mathrm{I}} t}{\phi x_{\mathrm{R}} y_{\mathrm{R}} h\left(1-s_{\mathrm{cw}}-s_{\mathrm{ro}}\right)}=\frac{q_{\mathrm{I}} t}{\phi x_{\mathrm{R}} y_{\mathrm{R}} h \Delta s} ;
$$

dependent variables:

$$
p_{\mathrm{cD}}=\frac{p_{\mathrm{c}} K_{\mathrm{row}} h}{q_{\mathrm{I}} \mu_{\mathrm{w}}}, p_{\mathrm{wD}}=\frac{p_{\mathrm{w}} K_{\mathrm{row}} h}{q_{\mathrm{I}} \mu_{\mathrm{w}}}, p_{\mathrm{oD}}=\frac{p_{\mathrm{o}} K_{\mathrm{row}} h}{q_{\mathrm{I}} \mu_{\mathrm{w}}}
$$

some other dimensionless parameters:

$$
\begin{gathered}
\mu_{\mathrm{oD}}=\frac{\mu_{\mathrm{o}}}{\mu_{\mathrm{w}}}, \quad \rho_{\mathrm{o} 0 \mathrm{D}}=\frac{\rho_{\mathrm{o} 0}}{\rho_{\mathrm{w} 0}}, \quad C_{\mathrm{oD}}=\frac{C_{\mathrm{o}} q_{\mathrm{I}} \mu_{\mathrm{w}}}{K_{\mathrm{row}} h}, \quad C_{\mathrm{wD}}=\frac{C_{\mathrm{w}} q_{\mathrm{I}} \mu_{\mathrm{w}}}{K_{\mathrm{row}} h}, \\
C_{\phi \mathrm{D}}=\frac{C_{\phi} q_{\mathrm{I}} \mu_{\mathrm{w}}}{K_{\mathrm{row}} h}, \quad p_{\mathrm{w} 0 \mathrm{D}}=\frac{p_{\mathrm{w} 0} K_{\mathrm{row}} h}{q_{\mathrm{I}} \mu_{\mathrm{w}}}, \quad p_{\mathrm{o} 0 \mathrm{D}}=\frac{p_{\mathrm{o} 0} K_{\mathrm{row}} h}{q_{\mathrm{l}} \mu_{\mathrm{w}}}, \\
p_{\mathrm{oiD}}=\frac{p_{\mathrm{oi}} K_{\mathrm{row}} h}{q_{\mathrm{I}} \mu_{\mathrm{w}}}, \quad p_{\mathrm{wfD}}=\frac{p_{\mathrm{wf}} K_{\mathrm{row}} h}{q_{\mathrm{I}} \mu_{\mathrm{w}}}, \quad r_{\mathrm{eoD}}=\frac{r_{\mathrm{eo}}}{x_{\mathrm{R}}}, \quad r_{\mathrm{oD}}=\frac{r_{\mathrm{o}}}{x_{\mathrm{R}}} ;
\end{gathered}
$$

and the normalized saturation and permeability:

$$
\bar{s}_{\mathrm{w}}=\frac{s_{\mathrm{w}}-s_{\mathrm{cw}}}{\Delta s}, \quad \bar{s}_{\mathrm{o}}=\frac{s_{\mathrm{o}}-s_{\mathrm{ro}}}{\Delta s}, \quad K_{\mathrm{oD}}=\frac{K_{\mathrm{o}}}{K_{\mathrm{cwo}}}, \quad K_{\mathrm{wD}}=\frac{K_{\mathrm{w}}}{K_{\mathrm{row}}},
$$


in which $x_{\mathrm{R}}, y_{\mathrm{R}}$ and $z_{\mathrm{R}}$ are reference lengths in three directions, respectively, $s_{\mathrm{ro}}$ and $s_{\mathrm{cw}}$ denote the residual oil saturation and the irreducible water saturation, respectively, $K_{\mathrm{cwo}}$ refers to the effective permeability of oil phase under the condition of the irreducible water saturation, $K_{\text {row }}$ the effective permeability of water phase under the condition of the residual oil saturation, and the subscript D means dimensionless parameter. Substituting all these variables into the governing equations, constitutive equations, and the initial and boundary conditions, we get a set of twenty-four scaling criteria of waterflooding system as follows:

$$
\begin{aligned}
& \frac{K_{\mathrm{cwo}}}{K_{\mathrm{row}}}, \frac{K_{\mathrm{o}}}{K_{\mathrm{cwo}}}, \frac{K_{\mathrm{w}}}{K_{\mathrm{row}}}, \frac{y_{\mathrm{R}}}{x_{\mathrm{R}}}, \frac{x_{\mathrm{R}}}{z_{\mathrm{R}}}, \frac{x_{\mathrm{p}}}{x_{\mathrm{R}}}, \frac{y_{\mathrm{p}}}{y_{\mathrm{R}}}, \frac{r_{\mathrm{eo}}}{x_{\mathrm{R}}}, \frac{r_{\mathrm{o}}}{x_{\mathrm{R}}}, \frac{s_{\mathrm{cw}}}{\Delta s}, \frac{s_{\mathrm{ro}}}{\Delta s}, \\
& \frac{s_{\mathrm{wi}}-s_{\mathrm{cw}}}{\Delta s}, \frac{\sigma \sqrt{\frac{\phi_{0}}{K}} \cos \theta K_{\mathrm{row}} h}{q_{\mathrm{I}} \mu_{\mathrm{w}}}, \frac{\mu_{\mathrm{o}}}{\mu_{\mathrm{w}}}, \frac{\rho_{\mathrm{o} 0}}{\rho_{\mathrm{w} 0}}, \frac{K_{\mathrm{row}} h}{q_{\mathrm{I}} \mu_{\mathrm{w}}} \rho_{\mathrm{w} 0} g z_{\mathrm{R}}, \frac{C_{\mathrm{o}} q_{\mathrm{I}} \mu_{\mathrm{w}}}{K_{\mathrm{row}} h}, \frac{C_{\mathrm{w}} q_{\mathrm{I}} \mu_{\mathrm{w}}}{K_{\mathrm{row}} h}, \\
& \frac{C_{\phi} q_{\mathrm{I}} \mu_{\mathrm{w}}}{K_{\mathrm{row}} h}, \frac{p_{\mathrm{w} 0} K_{\mathrm{row}} h}{q_{\mathrm{I}} \mu_{\mathrm{w}}}, \frac{p_{\mathrm{o} 0} K_{\mathrm{row}} h}{q_{\mathrm{I}} \mu_{\mathrm{w}}}, \frac{p_{\mathrm{wf}} K_{\mathrm{row}} h}{q_{\mathrm{I}} \mu_{\mathrm{w}}}, \frac{p_{\mathrm{oi}} K_{\mathrm{row}} h}{q_{\mathrm{I}} \mu_{\mathrm{w}}}, J\left(\bar{s}_{\mathrm{w}}\right) .
\end{aligned}
$$

They are defined as $\pi_{1}, \pi_{2}, \cdots, \pi_{24}$ hereafter. From the physical point of view, $\pi_{1}$ denotes the ratio of the oil permeability under the condition of the irreducible water saturation to the water permeability under the condition of the residual oil saturation, $\pi_{2}$ and $\pi_{3}$ the dimensionless permeability of water and oil phase, respectively, $\pi_{4}$ and $\pi_{5}$ the similarity of geometry, $\pi_{6}, \pi_{7}, \pi_{8}$ and $\pi_{9}$ the similarity of well position and well radius, $\pi_{10}$ the ratio of the irreducible water saturation to the mobile oil saturation, $\pi_{11}$ the ratio of the residual oil saturation to the mobile oil saturation, $\pi_{12}$ the reduced initial water saturation, $\pi_{13}$ the ratio of the capillary force to the reservoir pressure difference induced by the injection rate $q_{\mathrm{I}}, \pi_{14}$ and $\pi_{15}$ the ratios of the viscosity and density of water to oil respectively, $\pi_{16}$ the ratio of the gravitational force to the driving force, $\pi_{17}$, $\pi_{18}$ and $\pi_{19}$ the relative volume variation rations of oil, water and rock caused by the reservoir pressure difference induced by the injection rate $q_{I}$, respectively, $\pi_{20}, \pi_{21}, \pi_{22}$ and $\pi_{23}$ the respective ratios of the reference pressure of oil and water, the pressure of well bottom, and the initial pressure to the reservoir pressure difference and $\pi_{24}$ the capillary force function.

Consider a prototype with its parameters listed in Table 1. According to the above-derived scaling criteria, we may design a completely similar model with its parameters listed in Table 1, too. Performing numerical calculations for the model and the prototype, we obtain the oil recovery curves and water ratio at production well in both cases, as plotted in Figs. 4 and 5. The results of the model and the prototype are found in good agreement with each other, implying that the above-derived set of scaling criteria is complete. However, we can also see from Table 1 that a complete similarity means an 
Table 1 Parameters of the prototype and the corresponding scaled model

\begin{tabular}{cccccccccc}
\hline $\begin{array}{c}\text { Para- } \\
\text { meters }\end{array}$ & $\begin{array}{c}L \\
/ \mathrm{m}\end{array}$ & $\begin{array}{c}w \\
/ \mathrm{m}\end{array}$ & $\begin{array}{c}h \\
/ \mathrm{m}\end{array}$ & $\begin{array}{c}q_{I} \\
/ \mathrm{m}^{3} \mathrm{~s}^{-1}\end{array}$ & $\begin{array}{c}k_{\text {row }} \\
\times 10^{-12} / \mathrm{m}^{2}\end{array}$ & $\begin{array}{c}k_{\text {cwo }} \\
\times 10^{-12} / \mathrm{m}^{2}\end{array}$ & $\begin{array}{c}r_{\mathrm{o}} \\
/ \mathrm{m}\end{array}$ & $\begin{array}{c}C_{\mathrm{o}} \\
\times 10^{-10} / \mathrm{Pa}^{-1}\end{array}$ & $\begin{array}{c}C_{\mathrm{w}} \\
\times 10^{-10} / \mathrm{Pa}^{-1}\end{array}$ \\
\hline Prototype & 140 & 140 & 10 & $8 \times 10^{-3}$ & 0.37 & 0.78 & 0.1 & 8.0 & 5.0 \\
Model & 0.7 & 0.7 & 0.05 & $4 \times 10^{-5}$ & 0.407 & 0.858 & $5 \times 10^{-4}$ & 8.8 & 5.5 \\
\hline \hline $\begin{array}{c}\text { Para- } \\
\text { meters }\end{array}$ & $x_{\mathrm{p}}$ & $y_{\mathrm{p}}$ & $g$ & $C_{\phi}$ & $C_{\mathrm{m} 0}$ & $p_{\mathrm{o} 0}$ & $p_{\mathrm{wf}}$ & $p_{\mathrm{oi}}$ & $\sigma$ \\
\hline Prototype & 140 & 140 & 9.8 & 6.0 & 12.0 & 12.0 & 10.0 & 12.0 & 2.50 \\
Model & 0.7 & 0.7 & 1781 & 6.6 & 10.9 & 10.9 & 9.09 & 10.9 & 2.27 \\
\hline
\end{tabular}

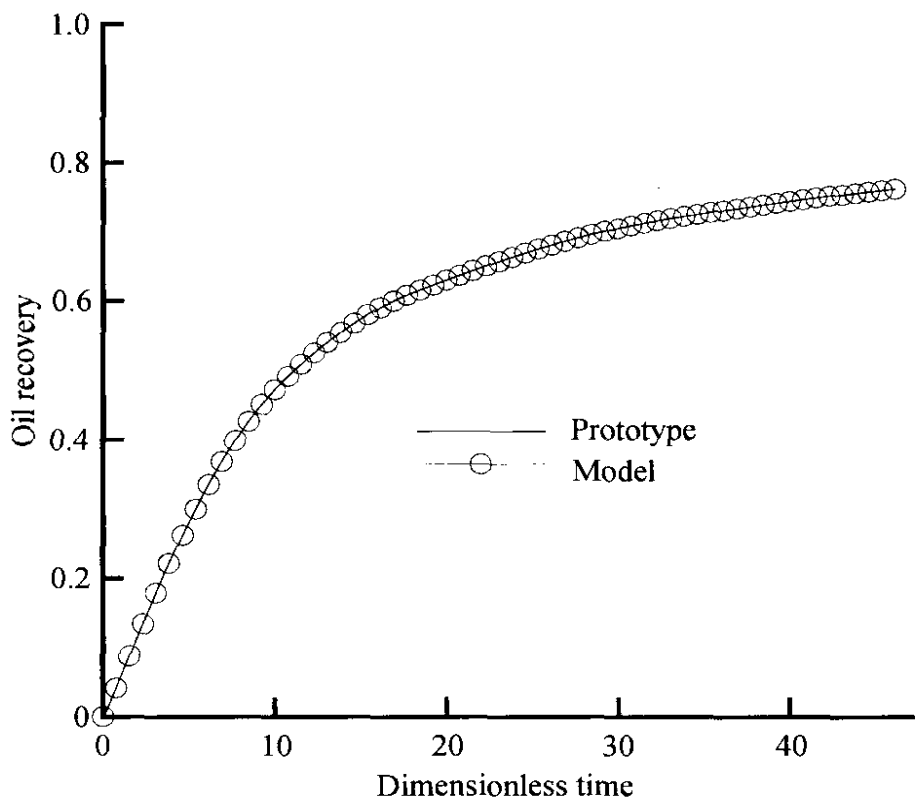

Fig. 4. Comparison of oil recovery between the prototype and the corresponding scaled model.

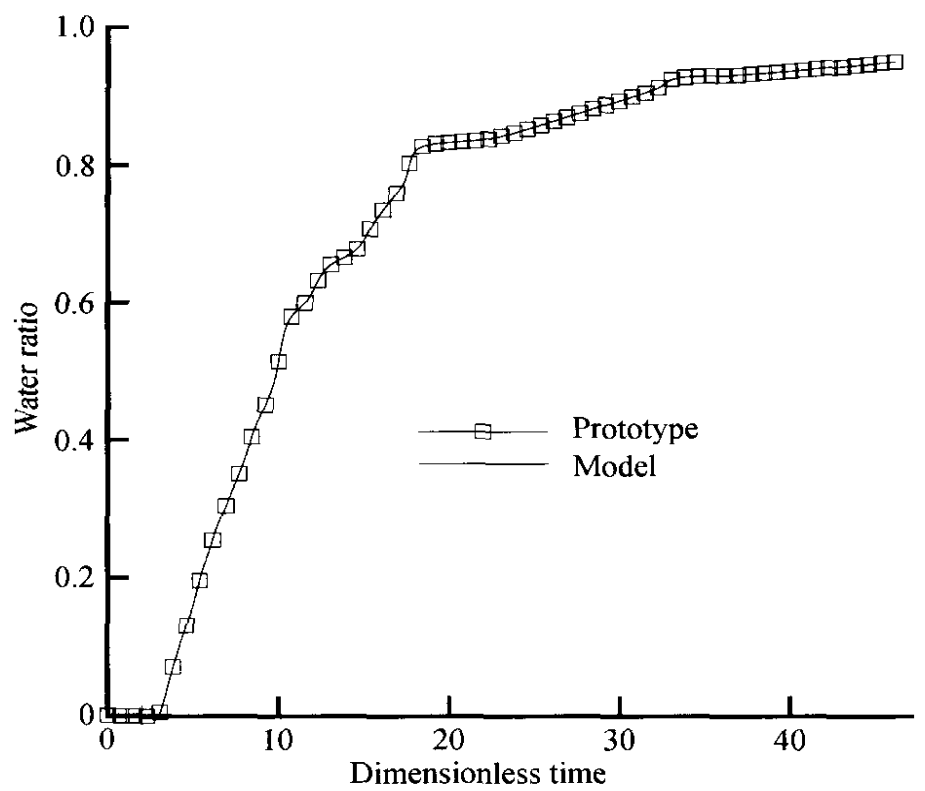

Fig. 5. Comparison of the water ratio between the prototype and the corresponding scaled model. 
impractically large gravitational acceleration. Therefore, we have to relax some of them in actual modeling. Nevertheless, we should identify which one is negligible at first.

\section{Sensitivity factors of dimensionless parameters}

According to the definition of the sensitivity factor aforementioned, we can find that sensitivity factor $S_{i}$ varies with dimensionless parameter $\pi_{i}$. For the prototype defined by the parameters listed in Table 1, the dimensionless parameters are evaluated as follows: $\pi_{1}=2.108, \pi_{4}=1, \pi_{5}=14, \pi_{6}=1, \pi_{7}=1, \pi_{9}=7.143 \times 10^{-4}, \pi_{10}=0.294, \pi_{11}=0.177, \pi_{12}$ $=0, \pi_{13}=5.781 \times 10^{-3}, \pi_{14}=5, \pi_{15}=0.8, \pi_{16}=4.533 \times 10^{-2}, \pi_{17}=1.730 \times 10^{-3}, \pi_{18}=$ $1.081 \times 10^{-3}, \pi_{19}=1.297 \times 10^{-3}, \pi_{20}=\pi_{21}=\pi_{23}=5.55, \pi_{22}=4.625, \pi_{2}$ and $\pi_{3}$ are normalized permeability of oil and water phases, and $\pi_{24}$ the capillary force function. Set the deviation coefficient $w_{i}$ of each dimensionless parameter to be $1 \%$ and $-1 \%$ respectively except for those representing the similarity of well locations, and keep the others identical between the model and the prototype. Then, we can readily derive the sensitivity factors as listed in Table 2.

Table 2 Sensitivity factors of dimensionless parameters

\begin{tabular}{cccccccc}
\hline$S_{i}$ & 1 & 2 & 3 & 4 & 5 & 9 & 10 \\
\hline$w_{i}=1 \%$ & $1.847 \times 10^{-1}$ & $1.845 \times 10^{-1}$ & $1.864 \times 10^{-1}$ & $3.713 \times 10^{-2}$ & $4.065 \times 10^{-3}$ & $1.148 \times 10^{-3}$ & $7.885 \times 10^{-3}$ \\
$w_{i}=-1 \%$ & $1.867 \times 10^{-1}$ & $1.870 \times 10^{-1}$ & $1.861 \times 10^{-1}$ & $3.461 \times 10^{-2}$ & $3.990 \times 10^{-3}$ & $1.167 \times 10^{-3}$ & $7.885 \times 10^{-3}$ \\
\hline \hline$S_{i}$ & 11 & 12 & 13 & 14 & 15 & 16 & 17 \\
\hline$w_{i}=1 \%$ & $9.974 \times 10^{-4}$ & $1.160 \times 10^{0}$ & $3.952 \times 10^{-4}$ & $1.848 \times 10^{-1}$ & $1.014 \times 10^{-1}$ & $4.742 \times 10^{-3}$ & $2.164 \times 10^{-3}$ \\
$w_{i}=-1 \%$ & $9.974 \times 10^{-4}$ & $1.260 \times 10^{0}$ & $3.952 \times 10^{-4}$ & $1.864 \times 10^{-1}$ & $1.012 \times 10^{-1}$ & $4.724 \times 10^{-3}$ & $2.164 \times 10^{-3}$ \\
\hline \hline$S_{i}$ & 18 & 19 & 20 & 21 & 22 & 23 & 24 \\
\hline$w_{i}=1 \%$ & $2.879 \times 10^{-3}$ & $3.049 \times 10^{-3}$ & $1.560 \times 10^{-3}$ & $1.272 \times 10^{-2}$ & $3.260 \times 10^{-2}$ & $2.755 \times 10^{-2}$ & $3.952 \times 10^{-4}$ \\
$w_{i}=-1 \%$ & $2.879 \times 10^{-3}$ & $3.048 \times 10^{-3}$ & $1.560 \times 10^{-3}$ & $1.274 \times 10^{-2}$ & $3.244 \times 10^{-2}$ & $2.751 \times 10^{-2}$ & $3.952 \times 10^{-4}$ \\
\hline
\end{tabular}

We can see from Table 2 that the order of sensitivity factors ranges from $10^{-4}$ to $10^{0}$. Apparently, the larger the sensitivity factor is, the more important the corresponding dimensionless parameter is. Hence, we can easily make a choice of dominant dimensionless parameters based on the numerical results. If we just reserve those parameters ranging from $10^{-1}$ to $10^{0}$, the scaling law looks like

$$
\frac{K_{\mathrm{cwo}}}{K_{\text {row }}}, \frac{K_{\mathrm{o}}}{K_{\mathrm{cwo}}}, \frac{K_{\mathrm{w}}}{K_{\text {row }}}, \frac{s_{\mathrm{wi}}-s_{\mathrm{cw}}}{\Delta s}, \frac{\mu_{\mathrm{o}}}{\mu_{\mathrm{w}}}, \frac{\rho_{\mathrm{o} 0}}{\rho_{\mathrm{w} 0}} .
$$

This implies that the ratio of the oil permeability under the condition of the irreducible water saturation to the water permeability under the condition of the residual oil saturation, the dimensionless permeability of water and oil, the density and viscosity ratios of water and oil, and the reduced initial water saturation are the most important parameters in water-flooding modeling and should be scaled precisely. These results exactly reflect what the most important and natural factors are in water-flooding flows. 
Among the attributes related to porous medium, the permeability exerts more influence on flows than others do. Among the properties of fluids, the density and viscosity are more important. Other factors, such as the gravitational force, the compressibility of water, oil and rock and the capillary force, may be relaxed if they contradict to the dominant ones. The reason is that the error induced by relaxing the secondary dimensionless parameters is far less than the dominant ones. We can also qualitatively draw these conclusions from the value of the dimensionless parameter itself. For example, $\pi_{13}$ means the relative importance between the capillary force and reservoir pressure difference induced by the injection rate $q_{\mathrm{I}}$. Its value implies that the capillary force is three orders less than that of the reservoir pressure difference under the condition of high permeability and large gradient of the driving force. $\pi_{16}$ represents the relative importance between the gravitational and the driving force. Its value of $4.533 \times 10^{-2}$ implies that the gravitational force is two orders less than that of the driving force for the case of not very thick reservoir and the small density difference between water and oil. Nevertheless, it is hard to qualify the dominance degree of all the dimensionless parameters in this way. Particularly, this qualitative analysis is of no help for quantifying the importance of the dimensionless parameters. This is exactly where the advantage of the proposed numerical approach lies.

\section{Conclusions}

In the present paper, the authors have proposed a numerical approach to analyze the sensitivity of each dimensionless parameter. A universally physical definition of the sensitivity factor is given to quantify the dependence degree of a target function on the dimensionless parameters. In regard to a water-flooding reservoir, we have derived a complete set of scaling criteria, which involves the effects of the gravitational force, the capillary force and the compressibility of water, oil and rock. By calculating the sensitivity factor numerically, we have further quantitatively identified the dominant dimensionless parameters. The results show that in the case of water-flooding reservoir, the order of the sensitivity factors ranges from $10^{-4}$ to $10^{0}$, and the most important scaling parameters are the ratio of the oil permeability under the condition of the irreducible water saturation to the water permeability under the condition of the residual oil saturation, the density and viscosity ratios between water and oil and the reduced initial water saturation. Very likely, the numerical optimization can be applied to other physical problems.

Acknowledgements This work was supported by the National 973 Project (Grant No. G1999022511), and the Innovation Project of Chinese Academy of Sciences.

\section{References}

1. Shen Pingping, Fundamental research on largely enhancing oil recovery, China Basic Science (in Chinese), 2003, 2: 9-14.

2. Sedov, L. I., Similarity and Dimensional Methods in Mechanics, New York: Academic Press, 1959.

3. Geertsma, J., Croes, G. A., Schwart, N., Theory of dimensionally scaled model of petroleum reservoir, Trans. AIME, 1956, 207: 118-127.

4. Kimber, K. D., Farouq, A. S. M., Puttagunta, V. R., New scaling criteria and their relative merits for steam 
recovery experiments, The Journal of Canadian Petroleum Technology, 1988, 27(4): 86-94.

5. Islam, M. R., Farouq, A. S. M., New scaling criteria for polymer, emulsion and foam flooding experiments, The Journal of Canadian Petroleum Technology, 1989, 28(4): 79-97.

6. Islam, M. R., Farouq, A. S. M., New scaling criteria for chemical flooding experiments, Journal of Canadian Petroleum Technology, 1990, 29(1): 29-36.

7. Shen Pingping, The theory and experiment of water and oil flow in porous media (in Chinese), Beijing: Oil Industry Press, 2000.

8. Pozzi, A. L., Blackwell, R. J., Design of laboratory models for study of miscible displacement, SPE, 1963, 4: $28-40$.

9. Kong Xiangyan, Chen Fenglei, Similarity criteria and physical simulation for water flooding (in Chinese), Petroleum Exploration and Development, 1997, 24(6): 56-60.

10. Khalid, A., Antonin, S., Petroleum Reservoir Simulation, London: Applied Science Publishers, 1979.

11. Kong Xiangyan, Advanced Mechanics of Fluids in Porous Media, Hefei: China Science \& Technology University Press, 1999. 